\title{
THE
}

$5-1-1990$

\section{Recursion Method in Quantum Spin Dynamics: The Art of Terminating a Continued Fraction}

\author{
V. S. Viswanath \\ Gerhard Müller \\ University of Rhode Island, gmuller@uri.edu
}

Follow this and additional works at: https://digitalcommons.uri.edu/phys_facpubs

Terms of Use

All rights reserved under copyright.

\section{Citation/Publisher Attribution}

V. S. Viswanath and Gerhard Müller. Recursion method in quantum spin dynamics: the art of terminating a continued fraction. J. Appl. Phys. 67 (1990), 5486-5488.

Available at: http://dx.doi.org/10.1063/1.345859

This Article is brought to you for free and open access by the Physics at DigitalCommons@URI. It has been accepted for inclusion in Physics Faculty Publications by an authorized administrator of DigitalCommons@URI. For more information, please contact digitalcommons-group@uri.edu. 


\title{
Recursion method in quantum spin dynamics: The art of terminating a continued fraction
}

\author{
V.S. Viswanath and Gerhard Muller \\ Department of Physics, The University of Rhode Island, Kingston, Rhode Island $02881-0817$
}

The results obtained from applications of the recursion method to quantum many-body dynamics can be greatly improved if an appropriate termination function is employed in the continued-fraction representation of the corresponding relaxation function. We present a general recipe for the construction and use of such termination functions along with two applications in spin dynamics. The method can be adapted to any other problem in quantum many-body dynamics.

The recursion method ${ }^{2}$ as applied to problems in the dynamics of quantum and classical Hamiltonian model systems $^{2,3}$ is essentially a modern version of the projection operator formalism designed by Mori and $\mathrm{Zwanzig}$, formulated in a language which makes it directly accessible to computational methods. However, the old problem of adequately terminating a continued fraction has resurfaced in recent dynamical studies which employ one or the other variant of the recursion method or the closely related moment method. ${ }^{4}$ The present work was motivated in particular by a study of Gagiiano and Balseiro, ${ }^{2}$ in which they use the recursion method for the calculation of the dynamic structure factor $S_{z z}(q, \omega)$ of the one-dimensional (ID) $s=1 / 2 X X Z$ model

$$
H=-\sum_{i=1}^{N}\left(S_{l}^{x} S_{i+1}^{x}+S_{i}^{y} S_{i+1}^{y}+\Delta S_{l}^{z} S_{i+1}^{z}\right)
$$

at zero temperature. The case $\Delta=0(X Y$ model) can be taken as a convenient test case, since the function $S_{z z}(q, \omega)$ is exactly known ${ }^{5}$ :

$$
\begin{aligned}
S_{z z}(q, \omega) \equiv & \int_{-\infty}^{+\infty} d t e^{i \omega t}\left\langle S_{q}^{z}(t) S_{-q}^{z}\right\rangle \\
= & 2\left[4 \sin ^{2}(q / 2)-\omega^{2}\right]^{-1 / 2} \Theta(\omega-\sin q) \\
& \times \Theta[2 \sin (q / 2)-\omega] .
\end{aligned}
$$

For the implementation of the recursion method at $T=0$, it is convenient to use the fermion representation of the $X X Z$ model; it has the advantage that the ground state has a simple structure in the limit $\Delta=0$ (noninteracting fermions). $S_{z z}(q, \omega)$ is then related to a fermion density correlation function.

The formulation of the recursion method used in Ref. 2 is based on an orthogonal expansion of the wave function

$$
|\dot{\psi}(t)\rangle \equiv G(t)|0\rangle=\sum_{k=0}^{\infty} D_{k}(t)\left|f_{k}\right\rangle,
$$

where $|0\rangle$ is the ground-state wave function of a given quantum model Hamiltonian $H$ and $G$ is the dynamical variable whose time-dependent correlation function

$$
\tilde{S}(\mathrm{t}) \equiv\left\langle 0\left|G(\mathrm{t}) G^{\dagger}\right| 0\right\rangle=\left\langle 0\left|G G^{\dagger}\right| 0\right\rangle D_{0}^{*}(-t)
$$

we wish to determine. The orthogonal vectors $\left|f_{k}\right\rangle$ are generated recursively via the Gram-Schmidt orthogonalization procedure with initial condition $\left|f_{0}\right\rangle=G|0\rangle,\left|f_{\ldots 1}\right\rangle \equiv 0$ :

$$
\begin{aligned}
& \left.\tilde{V}_{k+1}\right\rangle=\left(H-E_{0}\right)\left|f_{k}\right\rangle-\sigma_{k}\left|f_{k}\right\rangle-\delta_{k}\left|f_{k-1}\right\rangle, \\
& \sigma_{k}=\frac{\left\langle f_{k}\left|H-E_{0}\right| f_{k}\right\rangle}{\left\langle f_{k} \mid f_{k}\right\rangle}, \quad \delta_{k}=\frac{\left\langle f_{k} \mid f_{k}\right\rangle}{\left\langle f_{k-1} \mid f_{k-1}\right\rangle}
\end{aligned}
$$

It is assumed that the ground-state energy $E_{0}$ and wave function $|0\rangle$ have been determined as accurately as possible. The sequences of recurrents $\sigma_{k}, \delta_{k}$ then contain all information necessary to reconstruct the function $D_{0}(t)$ as follows. Insert the orthogonal expansion (2) into the Schrödinger equation, $i(\partial / \partial t)|\psi(t)\rangle=\left(H-E_{0}\right)|\psi(t)\rangle$, to obtain an infinite set of difference-differential equations for the functions $D_{k}(v)$, which, upon Fourier-Laplace transform,

$$
d_{k}(\xi) \equiv \int_{0}^{\infty} d t e^{i \zeta^{t}} D_{k}(t)
$$

can be solved for $d_{0}(\xi)$ in the continued-fraction representation

$$
d_{0}(\zeta)=\frac{i}{\zeta-\sigma_{0}-\frac{\delta_{1}}{\zeta-\sigma_{1}-\cdots}},
$$

and from which the frequency-dependent correlation function can be directly recovered:

$S(\omega) \equiv \int_{-\infty}^{+\infty} d t e^{i \omega t} \tilde{S}(t)=2\left\langle 0 ; G G^{\dagger} \mid 0\right\rangle \operatorname{Re} \lim _{\epsilon \rightarrow 0} d_{0}(\omega+i \epsilon)$.

Now consider the symmetrized correlation function

$$
\widetilde{\Phi}(t)=[\tilde{S}(t)+\tilde{S}(-t)] / 2=\left\langle 0\left|G G^{\dagger}\right| 0\right\rangle A_{0}(t)
$$

and its Fourier transform, the fiuctuation function $\Phi(\omega)$. $\widetilde{\Phi}(t)$ is equal to the real part of $\tilde{S}(t)$. In Fourier space we have (at $T=0) S(\omega)=2 \Phi(\omega) \Theta(\omega)$. The relaxation function $a_{0}(z)$, which is the Laplace transform of $A_{0}(t)$, can be expressed in a more concise continued-fraction representation than that of $d_{0}(\xi)$ :

$$
a_{0}(z)=\int_{0}^{\infty} d t e^{-z t} A_{0}(t)=\frac{1}{z+\frac{\Delta_{1}}{z+\frac{\Delta_{2}}{z+\cdots}}} .
$$

The associated spectral density is recovered as

$$
\Phi_{0}(\omega)=2 \operatorname{Re} \lim _{\epsilon \rightarrow 0} a_{0}(\epsilon-i \omega)
$$

and the fuctuation function as $\Phi(\omega)=\left\langle 0\left|G G^{\dagger}\right| 0\right\rangle \Phi_{0}(\omega)$. 
The first $n$ members of the sequence $\Delta_{k}$ are expressible in terms of the first $n$ pairs of the sequences $\sigma_{k}, \delta_{k}$ and vice versa by elementary transformation formulas. ${ }^{6}$ In a different formulation of the recursion method, ${ }^{3}$ the sequence $\Delta_{k}$ is obtained directly. In most practical applications of the recursion method, only a limited number of recurrents $\sigma_{k}, \delta_{k}$ or recurrents $\Delta_{k}$ can be calculated, which makes it necessary to terminate the continued fractions (3) or (4) artificially. The main goal of this report is to demonstrate a way to terminate (3) in such a way that the information content of the known $\Delta_{k}$ is exploited to the fullest extent without introducing artificial features.

For the dynamical structure factor $S_{z z}(q, \omega)$ at fixed wave numbers $q=3 \pi / 4$ and $\pi$ for the $X Y$ model at $T=0$, the first 13 recurrents $\Delta_{k}$ are displayed in the inset to Fig. 1 . They can be obtained directly from Lee's ${ }^{3}$ formulation of the recursion method or indirectly from the $\sigma_{k}, \delta_{k}$ in the approach taken by Gagliano and Balseiro. ${ }^{2}$ The relatively simple patterns revealed by the two $\Delta_{k}$ sequences can be interpreted as follows: The almost uniform sequence for $q=\pi$, which is $\Delta_{1}=2, \Delta_{2}=\Delta_{3}=\ldots=1$, is readily recognized as that of the Bessel function $A_{0}(t)=J_{0}(2 t)$ or its Fourier transform, the spectral density $\Phi_{0}(\omega)=2 / \sqrt{4-\omega^{2}}$; it is consistent (for $q=\pi$ ) with the exact result (1). For $q=3 \pi / 4$, the $\Delta_{2 k \sim 1}$ and the $\Delta_{2 k}$ converge (fairly rapidiy) to different values, $\Delta_{\infty}^{(o)}$ and $\Delta_{\infty}^{(e)}$, respectively, which is the unmistakable signature of a spectral density whose spectral weight is confined to a finite interval with a gap in the center, $\omega_{\min } \leqslant|\omega| \leqslant \omega_{\max }$, and with the two cut-off frequencies determined by

$$
\omega_{\max }=\sqrt{\Delta_{\infty}^{(e)}}+\sqrt{\Delta_{\infty}^{(0)}}, \quad \omega_{\min }=\sqrt{\Delta_{\infty}^{(e)}}-\sqrt{\Delta_{\infty}^{(0)}} .
$$

The next step in the procedure proposed here is to choose the simplest model spectral density which is consistent with these conditions,

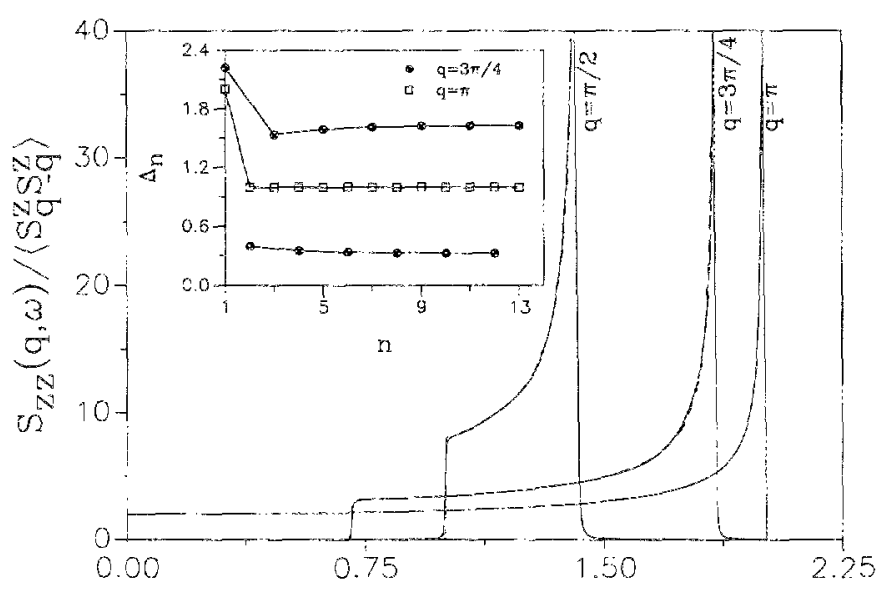

$\omega$

FIG. 1. Dynamic structare factor $S_{z: z}(q, \omega)$ (normalized by $\left.\left\langle S_{q} S^{2}{ }_{q}\right\rangle=q / 2 \pi\right)$ for fixed $q=n \pi / 4, n=2,3,4$, of the $1 \mathrm{D} s=1 / 2 X Y$ model at $T=0$. The full lines represent the result derived from the continued-fraction representation for $a_{0}(\epsilon \cdots i \omega)$ (with $\epsilon=0.001$ ) terminated at level $n=5$ as explained in the text. The dashed lines represent the exact result (1). The inset shows the recurrents $\Delta_{k}, k=1, \ldots, 13$, for $q=3 \pi / 4$ and $q=\pi$.

$$
\bar{\Phi}_{0}(\omega)=\frac{\pi}{\omega_{\max }-\omega_{\min }} \Theta\left(\omega-\omega_{\min }\right) \Theta\left(\omega_{\max }-\omega\right)
$$

and determine, via Hilbert transform, the associated model relaxation function

$\vec{a}_{0}(z)=\frac{1}{2 i\left(\omega_{\max }-\omega_{\min }\right)}\left(\ln \frac{z+i \omega_{\max }}{z-i \omega_{\max }}-\ln \frac{z+i \omega_{\min }}{z-i \omega_{\min }}\right)$.

A good approximation of the relaxation function $a_{0}\{z\}$, of which we only know a continued fraction (4) down to level $n$, is then constructed as follows: First expand the known model relaxation function $\bar{a}_{0}(z)$ into a continued fraction down to the nth level,

$$
\begin{aligned}
\bar{a}_{0}(z)=\frac{1}{z+\frac{\bar{\Delta}_{1}}{z+\cdots}} & \\
\cdots+\frac{\bar{\Delta}_{n-1}}{z+\bar{\Delta}_{n} \Gamma_{n}(z)} &
\end{aligned}
$$

which defines the nth-level termination function $\Gamma_{n}(z)$ along with the model recurrents $\bar{\Delta}_{k}, k=1, \ldots, n$. Then insert this termination function into the continued-fraction representation of the actual relaxation function $a_{0}(z)$, again at the $n$th level. In other words, approximate $a_{0}(z)$ by taking $\bar{a}_{0}(z)$ in the representation $(5)$ and replacing the model recurrents $\bar{\Delta}_{k}, k=1, \ldots, n$, with the known recurrents $\Delta_{k}$ of the dynamical quanticy under investigation. The termination can be performed at any level for which the true rechrrents are known, and it can be expected that the degree of approximation improves systematically as more model recurrents are replaced by exact ones.

In the case of the dynamic structure factor $S_{z z}(q, \omega)$ for the $X Y$ model, only few exact recurrents are needed to recover a good representation of the exact result (1). Our results shown in Fig. I for $S_{z z}(q, \omega)$ at $q=n \pi / 4, n=2,3,4$, are based on a continued fraction terminated by $\Gamma_{n}(z)$ at the level $n=5$. Comparison of these results with those of Fig. I in Ref. 2 demonstrates that there is much more information contained in the first few recurrents than can be retrieved from a finite continued fraction alone. The additional information is retrievable through the construction of a matching termination function.

For one more application of the method proposed here, consider the spin autocorrelation function $\left\langle S_{i}^{x}(t) S_{i}^{x}\right\rangle$ of the same model (the $X Y$ model at $T=0$ ), which can be rigorously expressed in terms of the solution of a nonlinear ODE as described in Refs. 8 and 9 . The associated spectrai density $\Phi_{0}^{x x}(\omega)$ was determined in Ref 9 on the basis of an exact long-time asymptotic expansion of $\left\langle S_{l}^{x}(t) S_{l}^{x}\right\rangle$ in conjunction with a precision numerical solution for short times. That spectral density, which is replotted in Fig. 2 (dashed line), has three singularities on the frequency range shown: an $\omega^{1 / 2}$ divergence at $\omega=0$, a logarithmic divergence at $\omega=1$, and an $\omega^{1 / 2}$ cusp at $\omega=2$.

The same nonlinear ODE from which these exact results were derived with much effort can be used to determine with relative ease a number of recurrents $\Delta_{k}$, from which the spectral density can be reconstructed approximately according to our scheme. These recurrents up to $\Delta_{13}$ are shown in the inset to Fig. 2. They have a linear average growth rate, 


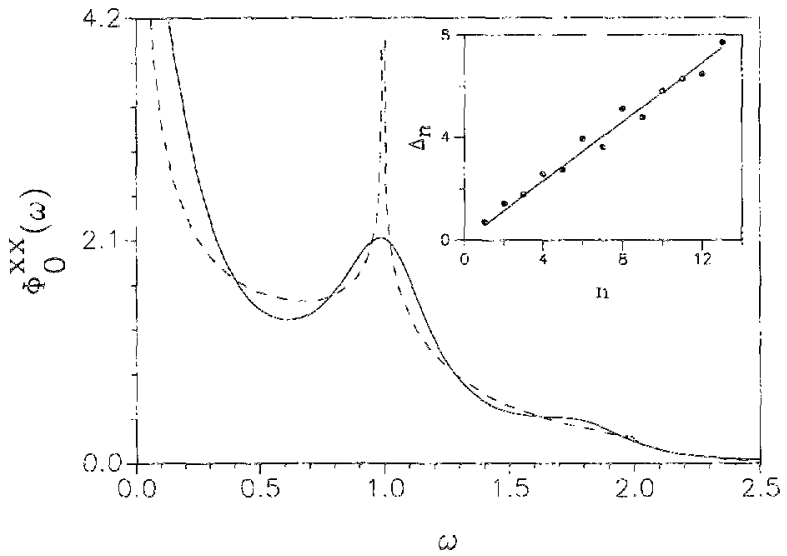

FIG. 2. Spectral density $\Phi_{0}^{x x}(\omega)$ for the spin autocorrelation function $\left\langle S_{1}^{x}(t) S_{i}^{x}\right\rangle$ of the $1 D S=1 / 2 X Y$ model at $T=0$. The full line represents the result derived from the continued-fraction representation for $a_{0}(\epsilon-i \omega)$ (with $\epsilon=0.001$ ) terminated at level $n:=13$ as described in the text. The recurrents $\Delta_{k}, k=1, \ldots, 13$, are shown in the inset along with the regression line $\Delta_{k}=0.574 k$. The dashed line represents the exact result from Ref. 9.

$\Delta_{k} \simeq 0.574 k$, with considerable scattering about that line. In this case we select the model spectral density $\bar{\Phi}_{0}^{x x}(\omega)$ by the requirement that its $\bar{\Delta}_{k}$ sequence reproduces the average behavior of the actual $\Delta_{k}$ sequence. This condition is satisfed by a pure Gaussian ${ }^{3}$ :

$$
\bar{\Phi}_{0}^{x x}(\omega)=\left(2 \sqrt{\pi} / \omega_{0}\right) \exp \left(-\omega^{2} / \omega_{0}^{2}\right), \quad \bar{\Delta}_{k}=k \omega_{0}^{2} / 2
$$

The associated model relaxation function,

$$
\bar{a}_{0}(z)=\left(\sqrt{\pi} / \omega_{0}\right) \exp \left(z^{2} / \omega_{0}^{2}\right) \operatorname{ercc}\left(z / \omega_{0}\right),
$$

with the parameter $\omega_{0}$ chosen to match the average growth rate of the $\Delta_{k}$ is then used to determine the $n$ th-level termination function $\Gamma_{n}(z){ }^{10}$ Inserted (at level $n=13$ ) into the continued-fraction representation of the relaxation function $a_{0}(z)$ yields the spectral density shown as solid line in Fig. 2. The approximate result reproduces all the major features of the exact spectral density at least qualitatively. Note in particular that our scherne of approximation has not produced any artificial features which may invite misinterpretation, a problem with which less sophisticated termination methods have been plagued almost inevitably.

In summary, we have outlined a practical method to terminate a continued fraction as it arises from the recursion method for two particular test cases. That procedure can be adapted to virtually any situation which one is likely to encounter in quantum many-body dynamics.

This work was supported in part by the U.S. National Science Foundation, Grant DMR-86-03036 and by a Grantin-Aid from the Council on Research of the University of Rhode Island. The numerical calculations were performed on the CRAY-2 at the National Center for Supercomputing Applications, University of Ilinois at Urbana-Champaign.

'D. G. Pettifor and D. L. Weaire, Eds., The Recursion Method and its Applications (Springer, New York, 1985).

${ }^{2}$ E. R. Gagliano and C. A. Balseiro, Phys. Rev. Lett. 59, 2999 (1987); Phys. Rev. B 38, 11766 (1988).

${ }^{3}$ M. H. Lec, Phys. Rev. B 26, 2547 (1982); J. Florencio and M. H. Lee, Phys. Rev. A 31, 3231 (1985); Phys. Rev. B 35, 1835 (1987).

${ }^{J}$. Oitmaa M. Plischke, and T. A. Winchester, Phys. Rev. 29, 1321 (1984).

${ }^{5}$ T. Niemeijer, Physica 36, 377 (1967); S. Katsura, T. Horiguchi, and M. Suzuki, Physica 46, $67(1970)$.

${ }^{\circ} \mathrm{G}$. Müller (unpublished).

${ }^{7}$ J. M. R. Roldan, H. M. McCoy, and J. H. H. Perk, Physica 136A, 255 (1986).

"B. M. McCoy, J. I. H. Perk, and R. E. Shrock, Nucl. Phys. B 220, 35 (1983).

"G. Müller and R. E. Shrock, Phys. Rev. 13 29, 288 (1984).

10 Note that our procedure is quite distinct from phenomenological approximations ( reviewed in $\mathbb{R}$ ef, 7 ) which employ one- or two-prarameter Gaussians for the termination function itself. Such approximations almost always result in a mismatch between the average slope of the $\Delta_{k}$ and the slope of the $\bar{\Delta}_{k}$ even if the average growth rate of $\Delta_{k}$ is, in fact, linear in $k$. This is, however, not generally the case. We have positive evidence for the occurrence of quadratic and cubic growth rates, which require entirely different types of termination functions. 